\title{
Structural Modifications of Multiwalled Carbon Nanotubes by Swift Heavy lons Irradiation
}

\author{
Keya Dharamvir ${ }^{1, a,{ }^{*}}$ Kiran Jeet $^{2,}$, , Chunsheng $\mathrm{Du}^{3, \mathrm{~b}}$, Ning $\mathrm{Pan}^{4, \mathrm{~b}}$ and \\ Vijay K. Jindal ${ }^{5, a}$ \\ aDepartment of Physics, Panjab University, Chandigarh-160014, India
}

${ }^{b}$ Nanomaterials in the Environment, Agriculture and Technology, University of California, Davis, USA

1'keya@pu.ac.in (corresponding author), ${ }^{2}$ bedikiran_80@yahoo.co.in, ${ }^{3} \mathrm{~cd} @ u c d a v i s . e d u$, 4npan@ucdavis.edu and 5jindal@pu.ac.in

Received: April 30, 2009; revised: July 6, 2009; accepted: August 24, 2009

Keywords: Carbon nanotubes; Ion irradiation; Raman spectroscopy; Defects.

\begin{abstract}
Thin film samples of multi-walled carbon nanotubes (MWCNTs) were irradiated with $120 \mathrm{MeV}$ gold ions. Transmission electron microscopy (TEM) images of the pristine and irradiated samples were obtained. TEM pictures show that in the irradiated sample, the CNTs are in general shorter and some have their inner cores filled, unlike in the pristine sample. We also find from these images that average inner and outer tube diameters change as a result of ion irradiation. The films were also characterized using Raman spectrometry. Modifications of the disorder mode (D mode) and the tangential mode ( $\mathrm{G}$ mode) under different irradiation fluences were studied in detail. As fluence increases, the MWCNTs first show damage, then healing under somewhat higher fluences and again amorphization under still higher fluence of ion irradiation.
\end{abstract}

\section{INTRODUCTION}

When any material is irradiated with a beam of energetic particles (ions or electrons) its mechanical, structural, and electrical properties are modified. High energy ions $(\mathrm{KeV}, \mathrm{MeV})$ have been used for the modification of materials while ions with very low energy $(\sim \mathrm{eV})$ have been used for thin film growth (epitaxy) [1, 2]. It is believed that swift heavy ions are responsible for significant electronic energy loss and may result in high pressure and temperature conditions in the target. Ion Irradiation using swift heavy ions produces a highly non equilibrium state which is never obtained under thermal equilibrium conditions. These unusual material processes may lead to new equilibrium condition resulting in novel material properties [3]. Recent studies have demonstrated that the irradiation of carbon nanotubes (CNT) with energetic particles can be used for nanoengineering processing, such as creating molecular junctions between nanotubes, making nanotubebased quantum dots and forming composite materials with enhanced mechanical properties [4]. Irradiation of CNTs with low-energy ion beams $(\mathrm{eV}$ to few $\mathrm{KeV}$ ) yield interesting structural transformations [5-9] such as welding, bending and straightening of nanotubes, creation of nanocapsules and, off course, amorphization.

So far little attention has been paid towards irradiation of CNTs by high energy ions. Misra et al. [10] used high energy (in the range of $100 \mathrm{MeV}$ ) gold $(\mathrm{Au})$ ions to study the structural damages of MWCNTs with nickel encapsulated inside. They found that irradiation produced localized defects on the tube walls, inducing amorphization of the encapsulated nickel nanorods. Kim et al. [11] observed formation of nano-compartments with bamboo like structure inside the MWCNTs and claim that the diameter of the MWCNTs expands upto 2.5 times after irradiation with $4 \mathrm{MeV} \mathrm{Cl}^{2+}$ ions at incident fluence of $3 \times 10^{16}$ ions $/ \mathrm{cm}^{2}$. Some studies have also been made using gamma rays and electrons. Hulman et al. [12] used gamma rays of energy $1.3 \mathrm{MeV}$ to irradiate single walled carbon nanotubes (SWCNT) as well as graphite and analyzed the results using Raman spectroscopy. They found that the irradiation generates defects in both the systems. A study based on Raman 
spectroscopy of the MWCNT under electron irradiation was done by Ritter et al. [13]. They used electrons of energy $1.8 \mathrm{MeV}$ to irradiate MWCNTs and reported both shift and change in intensity of vibrational modes under the influence of irradiation. Experimental and theoretical studies on structural evolution of MWCNTs under electron irradiation were undertaken by Banhart et al. [14]. However we did not find any work reporting the modification of MWCNTs using swift heavy ions (SHI). On the other hand, such studies have been done on thin films of $\mathrm{C}_{60}$ material, revealing important information about the structural modifications which take place mainly as a result of the energy deposited as $S_{\mathrm{e}}$ (electronic energy loss) [3, 15, 16 and references therein].

We attempt to explore these effects on CNTs by irradiating thin film samples of MWCNTs by swift heavy $\mathrm{Au}$ ions of energy $120 \mathrm{MeV}$. In the following sections we present the experimental details of sample preparation, irradiation and characterization results obtained from TEM and Raman spectroscopy.

\section{EXPERIMENTAL DETAILS}

\subsection{Sample preparation}

The nanotubes (MWCNTs) were synthesized by using chemical vapour deposition (CVD) method. The synthesis was conducted in a horizontal quartz tube in a furnace. The catalyst powder $\left(\mathrm{Fe} / \mathrm{Al}_{2} \mathrm{O}_{3}\right)$ for producing MWCNTs was placed in a ceramic boat which was then loaded into the tube furnace. Hydrogen gas was introduced into the furnace before it was heated up to $700^{\circ} \mathrm{C}$. The temperature was held for 30 minutes during which ethylene was introduced into the quartz tube. Ethylene supply was then shut off and the system was cooled down to room temperature. The obtained powder of $\mathrm{Fe} / \mathrm{Al}_{2} \mathrm{O}_{3} /$ MWCNTs was treated with a hydrogen fluoride (HF) aqueous solution with hydrochloride acid at room temperature successively to extract the nanotubes by dissolution of $\mathrm{Al}_{2} \mathrm{O}_{3}$ and iron particles. The nanotubes were then collected by filtration, washed with distilled water and dried. The purity of the MWNTs was about 95\%. Fig. 1 shows the TEM image of the purified MWCNTs which have an average outer diameter of the order of $15-20 \mathrm{~nm}$.

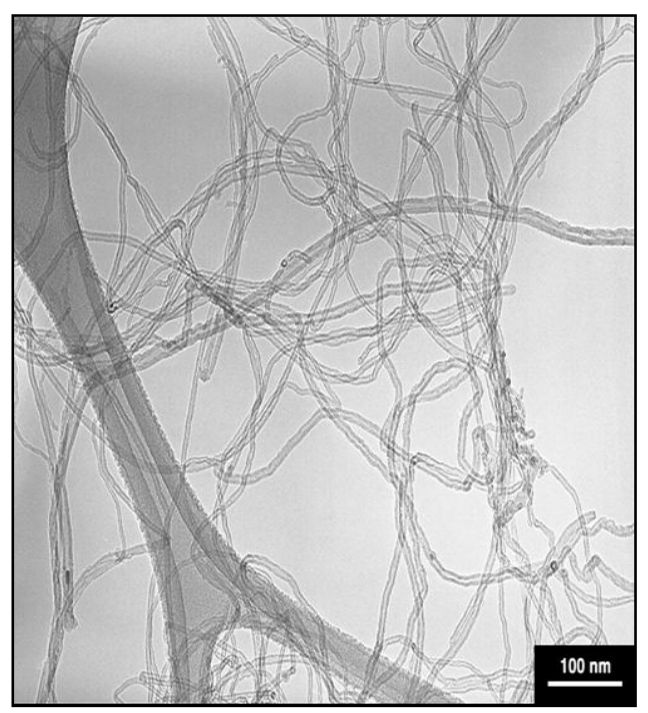

Fig. 1. Transmission electron microscopy (TEM) image of the purified MWCNTs before film formation.

We then prepared thin films samples of nanotubes on silicon wafers. Purified MWCNTs were refluxed with concentrated boiling nitric acid for 10 hours and then washed with distilled water followed by rinsing with isopropanol and drying in air at $60^{\circ} \mathrm{C}$. To prepare the colloidal suspension of carbon nanotubes in different solvents, predetermined amounts of refluxed carbon nanotubes were placed in a scintillation vial containing $10 \mathrm{ml}$ of dimethylformamide (DMF) and the vial was then capped and sonicated for 30 minutes. Colloidal suspensions were directly deposited on silicon 
wafers (N-type, resistivity: 1-5 $\Omega \mathrm{cm}$ ) and dried at room temperature followed by annealing at $160^{\circ} \mathrm{C}$ to remove any DMF which may have remained. The microstructures of these thin films were investigated by scanning electron microscopy (SEM) (FEI XL30-SFEG). The thickness of the films is found to be of the order of $4 \mu \mathrm{m}$.

\subsection{Irradiation and Characterisation}

The irradiation experiments were performed at Inter University Accelerator Centre (IUAC), New Delhi. We irradiated thin film samples of MWCNT with $\mathrm{Au}^{+9}$ ions of energy $120 \mathrm{MeV}$. Five samples were irradiated at fluences of $3 \times 10^{11}$ ions $/ \mathrm{cm}^{2}, 1 \times 10^{12}$ ions $/ \mathrm{cm}^{2}, 3 \times 10^{12}$ ions $/ \mathrm{cm}^{2}, 1 \times 10^{13}$ ions $/ \mathrm{cm}^{2}$ and $3 \times 10^{13}$ ions $/ \mathrm{cm}^{2}$. The ion beam was obtained from the 15 UD Pelletron accelator. The irradiated samples were studied using TEM and Raman spectroscopy. The TEM (Hitachi-7500) imaging was performed at Sophisticated Analytical Instrumentation Facility (SAIF), Panjab University, Chandigarh. The CNTs from the thin film were scrapped on to the TEM grid and the images were recorded. The Raman spectra of the thin films were measured using Raman spectrometer, Renishaw, model invia Raman Microscope. Raman data were recorded at room temperature with argon ion laser excitation $514 \mathrm{~nm}\left(5 \mathrm{~mW}\right.$ power) over a range $0-3200 \mathrm{~cm}^{-1}$ at IUAC.

\section{RESULTS AND DISCUSSION}

\subsection{TEM Results}

Fig. 2 shows the TEM pictures of two samples, one pristine and the other irradiated by Au ion of energy $120 \mathrm{MeV}$ at a fluence of $3 \times 10^{11}$ ions $/ \mathrm{cm}^{2}$. The qualitative changes taking place after irradiation are visible. We observe that the average length of CNTs is shorter in Fig. 2(b) and the outlines of tubes in this figure are not as clear as in the pristine sample shown in Fig. 2(a). The inset in Fig. 2(b) shows a tube whose interior has several dark spots. This characteristic was absent in all of the tubes in the pristine sample. We conjecture that these spots arise due to the presence of amorphous carbon produced after the breaking of the innermost walls of the tubes as a result of irradiation. Intense irradiation is expected to disturb the atomic position in all tubes surrounding the core tube and subsequently cause its collapse. Similar behavior of collapse of innermost wall under irradiation is discussed in the works of Banhart et al. [14] and Krasheninnikov et al. [17]. The innermost broken wall turns into a-C and the debris remain inside the tube blocking the channel. We note that only a few of the CNTs show this character, whereas most of the other tubes have a hazy outline for the inner wall.
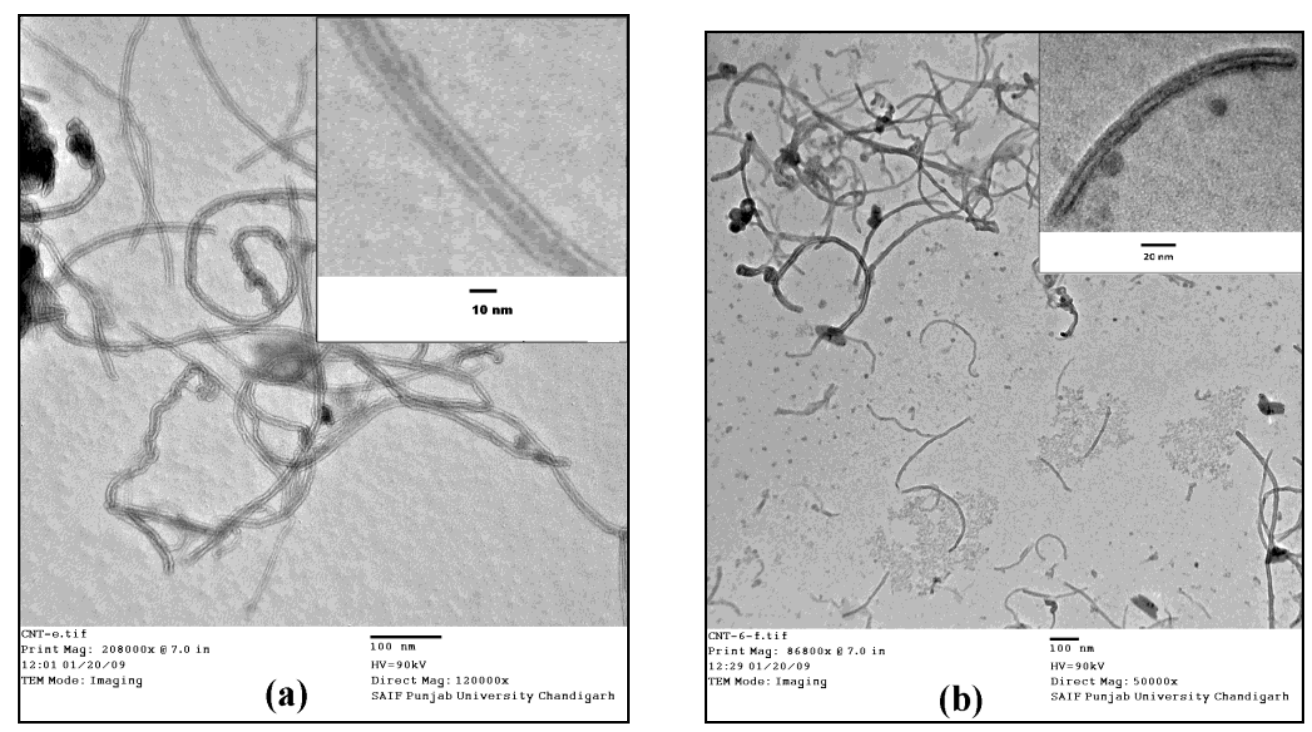

Fig. 2. TEM images of MWCNT samples (a) Pristine and (b) Irradiated (at fluence $3 \times 10^{11}$ ions $/ \mathrm{cm}^{2}$ ). Inset in these figures show magnified views of single MWCNTs. 
We measure the inner and outer diameters of the tubes in both pristine and irradiated samples. The statistical analysis of this data is presented in figures 3 (a) and 3 (b). The average diameter values are given in table 1. These numbers show that the irradiated MWCNTs, on the average, have shed quiet a few of the outer walls (pl. see estimate given at the end of sec. 3.2.2). The inner diameters of the tubes on the other hand show no significant change as far as their distribution is concerned (see Fig. 3(a)) although the average value has become larger. In other words, a smaller proportion of MWCNTs have lost their innermost wall. These TEM results give the preliminary information that the MWCNTs lose their walls both from inside and outside.

\begin{tabular}{|l|c|c|}
\hline \multicolumn{1}{|c|}{ Diameter } & $\begin{array}{c}\text { Pristine sample } \\
{[\mathrm{nm}]}\end{array}$ & $\begin{array}{c}\text { Irradiated Sample } \\
{[\mathrm{nm}]}\end{array}$ \\
\hline Inner diameter & $4.6 \pm 0.5 \mathrm{~nm}$ & $4.8 \pm 0.5 \mathrm{~nm}$ \\
\hline Outer diameter & $13.54 \pm 0.5 \mathrm{~nm}$ & $10.86 \pm 0.5 \mathrm{~nm}$ \\
\hline
\end{tabular}

Table I. Average value of diameters (in nm) of pristine and irradiated MWCNTs
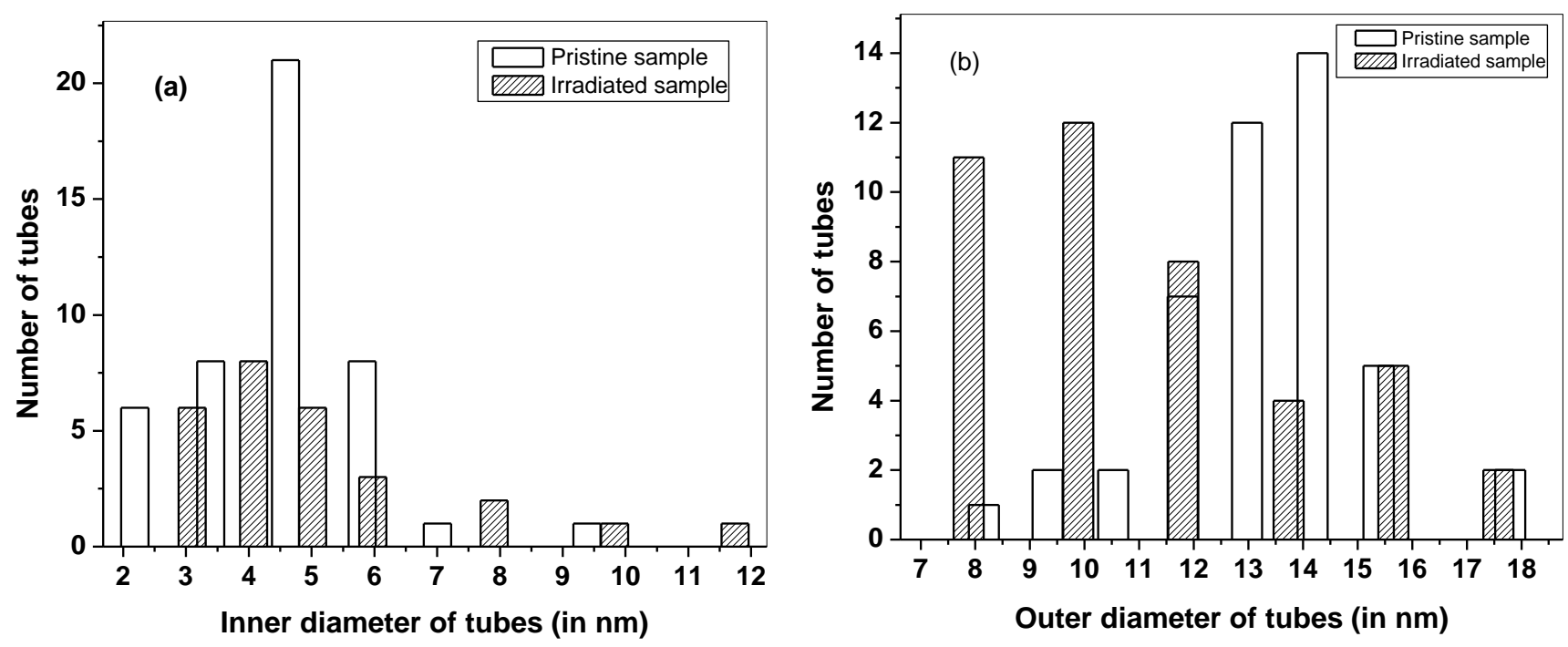

Fig. 3. Diameter distribution of pristine and irradiated MWCNTs (a) Inner diameter (b) Outer diameter.

\subsection{Raman Results}

\subsubsection{Raman modes of the pristine sample}

Raman spectra covering the entire frequency range from 100 to $3200 \mathrm{~cm}^{-1}$ are recorded for all the samples. The results for the pristine sample over the entire range are shown in Fig. 4 (a). In the low frequency part of the Raman spectrum, a scattering peak appears at the position $292 \mathrm{~cm}^{-1}$. Normally one expects a radial breathing mode (RBM) in this region. The RBM is an important Raman feature which corresponds to coherent vibrations of the $\mathrm{C}$ atoms in the radial direction. This unique feature, peculiar to carbon nanotubes, occurs in a frequency range of 120 to $350 \mathrm{~cm}^{-1}$. If the RBM frequency $\left(\omega_{\mathrm{RBM}}\right)$ of a SWCNT is known, its diameter can be calculated using the expression $\omega_{\mathrm{RBM}}=\mathrm{A} / \mathrm{d}_{\mathrm{t}}+\mathrm{B}$, where ' $A$ ' and ' $B$ ' are determined experimentally $\left(A=234 \mathrm{~cm}^{-1}\right.$ and $\left.B=10 \mathrm{~cm}^{-1}\right)$ [18-20]. In case 
of MWCNTs, the RBM, if detected, corresponds to its innermost wall [21]. However, in our work, we do not attribute the peak at $292 \mathrm{~cm}^{-1}$ to RBM, because of the following two reasons. Firstly, in the irradiated samples no signal persists in the vicinity of this peak. Secondly, the diameter calculated using this frequency in the above expression, does not agree with the internal diameter of our MWCNTs. The value of internal diameter as calculated from RBM comes out to be $0.86 \mathrm{~nm}$ whereas the average internal diameter as observed from TEM for the pristine sample is $4.6 \mathrm{~nm}$. So this peak appears to be originating from the background effects.

In the frequency range 1000-2000 $\mathrm{cm}^{-1}$, characteristic first order Raman modes are observed. The peak at $1354.8 \mathrm{~cm}^{-1}$ is identified as the defect induced D peak which generally arises in $s p^{2}$ carbon samples containing pores, impurities and other symmetry breaking defects [11]. Another prominent peak is observed at $1586.4 \mathrm{~cm}^{-1}$ and is identified as the $E_{2 g}$ mode, also known as the G peak. Fig. 4 (a), which presents a detailed spectrum of the pristine sample from fig. 4 (b), shows the splitting of the $G$ peak into two different peaks, $G^{-}$at $1586.4 \mathrm{~cm}^{-1}$ and $G^{+}$at $1621.3 \mathrm{~cm}^{-1}$. The $\mathrm{G}^{-}$peak is relatively high in intensity compared to the $\mathrm{G}^{+}$peak, not only in the pristine sample, but also in the irradiated ones. However in the work by Costa et al. [22] the $\mathrm{G}^{+}$peak is higher than $\mathrm{G}^{-}$. The lower frequency component $\mathrm{G}^{-}$, which is associated with vibrations along the circumference of a CNT, depends upon the diameter of the nanotube while the high frequency component $\mathrm{G}^{+}$, which is associated with vibrations along the nanotube axis [22], is independent of the diameter. The $\mathrm{G}^{-}-\mathrm{G}^{+}$ splitting is large for small diameter SWCNTs but the corresponding splitting in MWCNT is smeared out as there is a diameter distribution within the individual MWCNT [22]. Comparing the intensities of the first order Raman modes, it is observed that generally the intensity of the D peak is smaller than that of the G peak in SWCNT samples, but in case of MWCNT samples their intensities are almost equal. This has been explained taking into account the fact that the crosssection of the MWCNT involves a large number of diameter values [13]. In our case, the pristine sample is found to have higher value of D peak $\left(1354.8 \mathrm{~cm}^{-1)}\right.$ intensity as compared with $\mathrm{G}$ peak $\left(1586.3 \mathrm{~cm}^{-1}\right)$. The reason for high intensity of the $\mathrm{D}$ peak can be attributed to the fictionalization of MWCNTs done during the preparation of thin films. The fictionalization involves acid treatment of CNTs and is likely to cause the formation of defects on the walls of the tube and thus increases the intensity of the D peak.
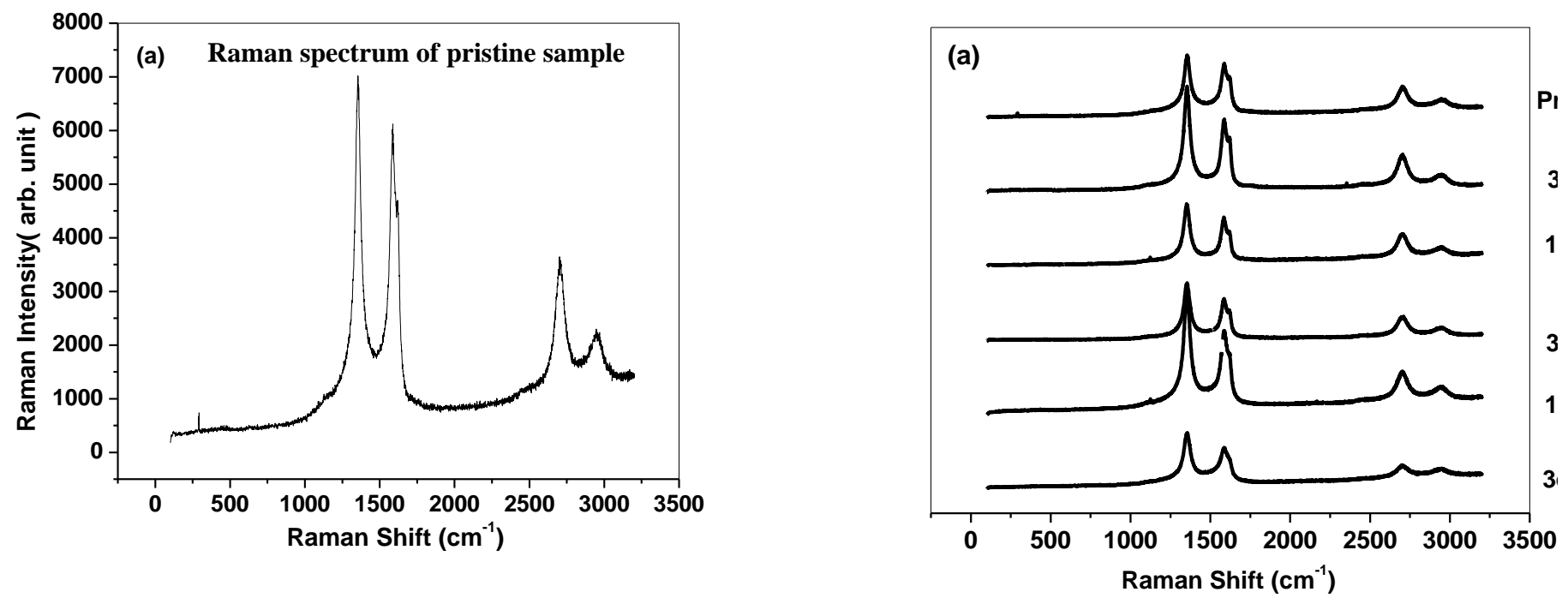

Fig. 4. Raman spectrum in the frequency range $100-3200 \mathrm{~cm}^{-1}$ (a) pristine sample and (b) irradiated samples at various fluences ranging from $3 \times 10^{11}$ ion $/ \mathrm{cm}^{2}$ to $3 \times 10^{13}$ ions $/ \mathrm{cm}^{2}$ (the curves have been shifted for the sake of clarity). 
In the second order Raman spectrum two peaks are observed in the frequency range 2000-3000 $\mathrm{cm}^{-1}$. The first peak appearing at $2706.2 \mathrm{~cm}^{-1}$ corresponds to the overtone of the peak at $1355.8 \mathrm{~cm}^{-1}$ (D mode) and the second peak appearing at $2945 \mathrm{~cm}^{-1}$ is identified as the combination of two strong Raman modes at $1350 \mathrm{~cm}^{-1}$ and $1620 \mathrm{~cm}^{-1}$ [23].

\subsubsection{Raman modes of irradiated samples}

We now present the Raman spectra of all the irradiated samples (from irradiation fluence $3 \times 10^{11}$ to $3 \times 10^{13}$ ions $/ \mathrm{cm}^{2}$ ) along with the pristine sample in the frequency range $0-3200 \mathrm{~cm}^{-1}$ (see Fig. 4(b)). The D and G peaks, along with the second order Raman modes, are found in all five irradiated samples. In order to have a quantitative estimate of the changes in intensities of these peaks under the effect of irradiation, we fit both the peaks (D and G) with several Lorentzians (Fig.5). The study based on Lorentzian fit of these samples shows that with increase in ion fluence values, the intensities of the $D$ and $G$ peaks vary whereas their positions remain practically unaltered. Also, the area under the D peak seems to fluctuate under varying irradiation fluence. Similar behavior of rise and fall of the D peak intensity is seen in other works also $[12,13]$.
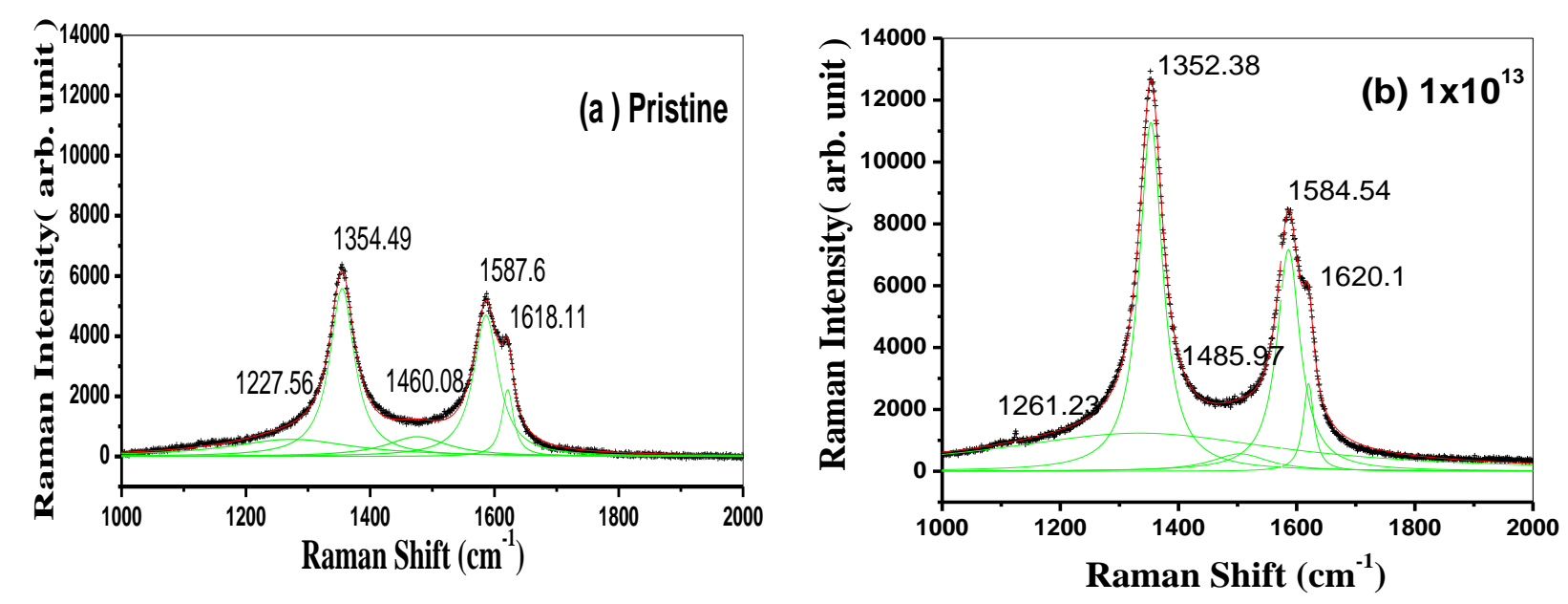

Fig. 5. High frequency Raman spectra of MWCNT samples along with Lorentzian fits for both $D$ and $G$ peaks (a) Pristine sample and (b) Irradiated samples at irradiation fluence $1 \times 10^{13}$ ions $/ \mathrm{cm}^{2}$.

To further study the modifications occurring due to irradiation, we plot the variation of disorder parameter as a function of ion fluence (Fig. 6). The disorder parameter, which is defined as the ratio of integral intensities of $D$ and $G$ peaks (I (D)/I (G)), generally expresses the degree of amorphization of the system. The inset in this figure shows the variation of integral intensities of $D$ and $G$ modes normalized with respect to the second order $D$ mode. The second order $D$ mode has been used for normalizing the D and $G$ intensities because it is found [24] that its intensity is independent of the defect concentration in the sample.

From Fig. 6, we observe that at the minimal fluence $3 \times 10^{11}$ ions $/ \mathrm{cm}^{2}$, the disorder parameter rises from the value of the pristine sample. It then falls at the next irradiation fluence $\left(1 \times 10^{12}\right.$ ions $/ \mathrm{cm}^{2}$ ). Thereafter it keeps rising slowly but never attains the high value as obtained at $3 \times 10^{11}$ ions $/ \mathrm{cm}^{2}$. The rise in the intensity of the disorder parameter at first incident fluence indicates the production of defects due to irradiation. The incident gold ion displaces carbon atoms from the MWCNTs causing these defects. According to theoretical calculations, the threshold energy required to displace a carbon atom from the surface of any carbon nanostructure ranges from $12 \mathrm{eV}$ to $80 \mathrm{eV}$ [13]. The gold ion with energy $120 \mathrm{MeV}$ is able to cause such defects 


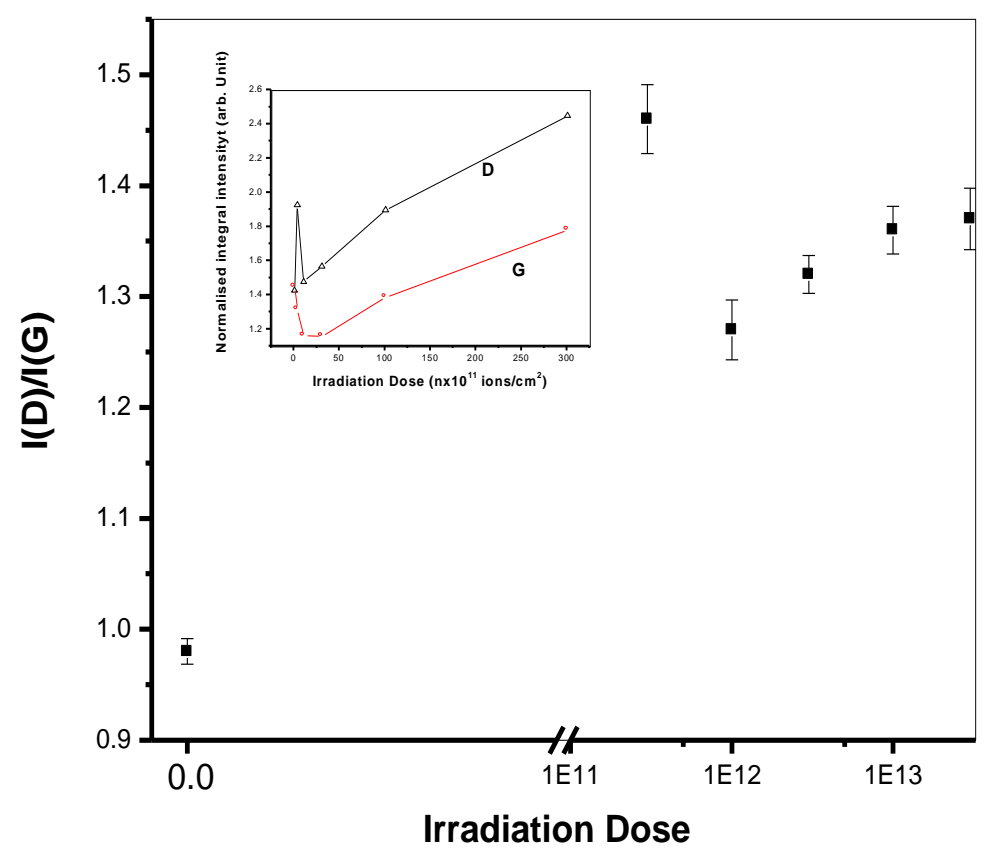

Fig. 6. Variation of disorder parameter (intensity ratio, $D$ to $G$ peak) as function of irradiation fluence $n \times 10^{11}$ ions $/ \mathrm{cm}^{2}$ (where value of $\mathrm{n}=3,10,30,100$ and 300 ). Inset shows variation of the respective individual peaks integrated intensities, normalized with respect to $D^{*}$ peak.

It is pertinent here to compare our work with that of Kumar et al. [25], where a similar experiment was performed using $60 \mathrm{MeV} \mathrm{Ni}$ ions for which electron energy loss parameter, $S_{\mathrm{e}}=$ $527.7 \mathrm{eV} / \mathrm{A}$. The I(D)/ I(G) ratio in that work shows steady rise (except at the low dose below $4 \mathrm{x}$ $10^{11}$ ions $/ \mathrm{cm}^{2}$ ) till the highest fluence, $1 \times 10^{13}$ ions $/ \mathrm{cm}^{2}$. This fluence corresponds to $\Phi$ (we define $\Phi=$ Se $x$ fluence $)=0.522 \mathrm{eV} / \mathrm{A}^{3}$. This quantity represents the total electronic energy deposited and is a rough measure of the damage caused in the material, which we expect to be reflected by the $\mathrm{I}(\mathrm{D}) / \mathrm{I}(\mathrm{G})$ ratio. At the first fluence used in our experiment $\left(\mathrm{S}_{\mathrm{e}}=1071 \mathrm{eV} / \mathrm{A}\right.$ and fluence $=3 \times 10^{11}$ ions $\left./ \mathrm{cm}^{2}\right) \Phi=0.032 \mathrm{eV} / \mathrm{A}^{3}$ and at our next higher fluence $\Phi=0.1 \mathrm{eV} / \mathrm{A}^{3}$ which is nearly the same amount as the highest energy deposited in Kumar et al work. Thus, if we had used smaller steps of irradiation doses, we would have seen the curve in Fig. 6 slowly rising between the fluences of 0 and $1 \times 10^{11}$ ions $/ \mathrm{cm}^{2}$.

After the initial damage, further fluence causes overall ordering of the system (fall in D/G ratio) which could be attributed to annealing. We suggest in the following paragraphs two mechanisms for this annealing. Further higher fluences are seen to increase the disorder parameter indicating overall damage or amorphization in the system. These two processes, annealing and amorphization, can be understood as competing processes taking place at all fluences.

One possible mechanism of the annealing process could be via rearrangement of the bonds. This can be explained on the basis of ion solid interaction as in the work of Kumar et al. [25]. When an energetic ion passes through the materials it heats up the material along the ion track by transferring its $S_{\mathrm{e}}$, leading to electron phonon coupling, which then shakes up the atoms raising the probability of rejoining or ordering of bonds. The healing of bonds makes the MWCNTs more ordered or purified.

The other reason for the drop in disorder parameter with irradiation could be due to a mechanism of seam opening. The knocking-off of atoms from the outermost surface of CNTs leads to several types of damages including those in the form of seams. Similar result of seam formation due to the 
effect of electron irradiation on MWCNTs is reported by Ritter et al. [13]. Once the seam is formed on the CNT surface, further irradiation degrades the quality of the seam and also reduces the binding of the defected layer with its next neighboring layer, which further helps in breaking of the seam. Therefore the tube unfolds itself into a graphite sheet along the seam. Similar type of unzipping of MWCNTs using chemical treatment has been reported recently [26-29]. As a result of unfolding of the defected tube, the next clean wall gets exposed to irradiation. Such a phenomenon should result in decrease of disorder parameter.

The trend of individual $D$ and $G$ peaks (inset in Fig. 6) in this experiment is unlike those found in other $\mathrm{sp}^{2}$ carbon systems $[12,30]$. The sharp peak seen in the D-curve at relatively low fluence is a new feature peculiar to this system. This leads us to believe in the phenomenon of seam opening which can only take place in a MWCNT system.

The result of Raman spectroscopy about the peeling of the outer layer of MWCNTs under the effect of ion irradiation is consistent with the TEM results, where we find that the average outer diameter of the tubes reduces by about $2.68 \mathrm{~nm}$ (which is $28.6 \AA$, whereas the separation between two adjacent layers of CNTs is $0.34 \mathrm{~nm}$; and $26.8 / 6.8=3.9$ ) which corresponds to approximately four walls in a CNT. Therefore we conclude that the incident ions of gold of energy $120 \mathrm{MeV}$ and fluence value $3 \times 10^{11}$ ions $/ \mathrm{cm}^{2}$ are capable of peeling four outer layers of MWCNTs.

\section{SUMMARY AND CONCLUSION}

We have studied the effect of irradiation of gold ion beam of energy $120 \mathrm{MeV}$ on thin film samples of MWCNT. From the TEM data we find that on average the outer diameters of the irradiated tubes are reduced while their inner diameters increase on the average, indicating that MWCNTs lose their walls both from inner as well as outer surfaces as a result of irradiation. The Raman results were studied through the modification of $\mathrm{D}$ and $\mathrm{G}$ peak. The behavior of these peaks shows that at a low value of ion fluence (higher than that causing the initial high damage), the condition of seam formation or healing of MWCNTs becomes more favorable. As we proceed to higher fluences, amorphization of the system dominates.

It is now apparent that our data is scanty because of limited fluence exposures. However this preliminary investigation indicates that it is possible to systematically peel off the outermost wall of MWCNTs taking incident beam at controlled values of ion fluence. Further work at smaller steps of fluence will be useful to strengthen this belief and quantify the fluence values which can be used to systematically destroy outer layers.

The sequences of these various types of damage and annealing can be studied well by computer simulations apart from measurements. We propose to further carry out this work on SHI irradiation of MWCNTs taking both these aspects into account.

\section{ACKNOWLEDGMENTS}

KD and KJ acknowledge a discussion with Prof. O. N. Srivastava, Banaras Hindu University, India, which helped clarify some of the issues. KJ acknowledges financial support from University Grant Commission (UGC), India. 


\section{REFERENCES}

[1] B. Q. Wei, J. D. Arcy-Gall, P. M. Ajayan and G. Ramanath, Appl. Phys. Lett. 83 (2003) 35813583.

[2] B. Ni, R. Andrews, D. Jacques, D. Qian, M. B. J. Wijesundara, Y. Choi, L. Hanley, and S. B. Sinnott, J. Phys. Chem. B 105 (2001) 12719-12725.

[3] N. Bajwa, A. Ingale, D.K. Avasthi, R. Kumar, A. Tripathi, K. Dharamvir, and V.K. Jindal, Radiation measurement 36 (2003) 737-740.

[4] A.V. Krasheninnikov, and K. Nordlund, Nuclear Instruments and Methods in Physics Research B 216 (2004) 355-366.

[5] Z. Wang, L. Yu, W. Zhang, Y. Ding, Y. Li, J. Han, Z. Zhu, H. Xu, G. He, Y. Chen, and G. Hu, Phys. Lett. A 324 (2004) 321-325.

[6] Y. Kurokawa, Y. Ohno, S. Kishimoto, T. Okazaki, H. Shinohara, and T. Mizutani, J. Appl. Phys. 43 (2004) (Japan) 5669-5670.

[7] Y. Joon Jung, Y. Homma, R. Vajtai, Y. Kobayashi, T. Ogino, and P. M. Ajayan, Nano letters 4, (2004) 1109-1113.

[8] M. Suzuki, K. Ishibashi, K. Toratani, D. Tsuya, and Y. Aoyagi, Appl. Phys. Lett. 81 (2002) 2273-2275.

[9] K. Ishibashi, D. Tsuya, M. Suzuki and Y. Aoyagi, Appl. Phys. Lett. 82 (2003) 3307-3309.

[10]. A. Misra, P. K. Tyagi, M. K. Singh, D.S. Misra, J. Ghatak, P. V. Satyam, and D. K. Avasthi, Diamond and Related Materials 15 (2006) 300-303.

[11] H. M. Kim, H. S. Kim, S. K. Park, J. Joo, T. J. Lee, and C. J. Lee, J. Appl. Phys. 97 (2005) 026103-1 - 026103-3.

[12] M. Hulman, V. Skakalova, S. Roth, and H. Kuzmany, J. Appl. Phys. 98 (2005) 024311-1 024311-5.

[13] U. Ritter, P. Scharff, C. Siegmund, O.P. Dmytrenko, N. P. Kulish, Y. I. Prylutskyy, N. M. Belyi, V. A. Gubanov, L. I. Komarova, S. V. Lizunova, V. G. Poroshin, V. V. Shlapatskayan, and H. Bernas, Carbon 44 (2006) 2694-2700.

[14] F. Banhart, J. X. Li, and A. V. Krasheninnikov, Phys. Rev. B 71 (2005) 241408-1 -241408-4.

[15] N. Bajwa, K. Dharamvir, and V. K. Jindal A. Ingale, D.K Avasthi, R. Kumar, A. Tripathi, J. Appl. Phys. 94 (2003) 326-333.

[16] N. Bajwa, A. Ingale, D. K Avasthi, R. Kumar, A. Tripathi, K. Dharamvir, and V.K. Jindal, J. Appl. Phys. 104 (2008) 054306-1 - 054306-13.

[17] A. V. Krasheninnikov, F. Banhart, J. X. Li, A. Foster, and R. Nieminen, Phys. Rev. B 72 (2005) 125428-1 -125428-6.

[18] M.S. Dresselhaus, G. Dresselhaus, R. Saito, and A. Jorio, Physics Reports, 409:2, (2005), 4799.

[19] A. Jorio, R. Saito, J. H. Hafner, C. M. Lieber, M. Hunter, T. McClure, G. Dresselhaus, and M. S. Dresselhaus, Phys. Rev. Lett. 86, 1118 - 1121

[20] M. Milnera, J. Kürti, M. Hulman, and H. Kuzmany, Phys. Rev. Lett. 84, (2000), 1324 - 1327.

[21] X. Zhao, Y. Ando, L. -C. Qin, H. Kataura, Y. Maniwa, and R. Saito, Physica B: Condensed Matter, 323:1-4, (2002), 265-266.

[22] S. Costa, E. B. Palen, M. Kruszynska, A. Bachmatiuk, and R J. Kalenczuk, Materials SciencePoland, 26/2 (2008) $433-441$. 
[23] R.J. Nemanich, and S.A. Solin, Phys. Rev. B 20 (1979) 392-401.

[24] J. Maultzsch, S. Reich, C. Thomsen, S. Webster, R. Czerw, D. L. Carroll S. M. C. Vieira, P. R. Birkett, and C. A. Rego, Appl. Phys. Lett. 81, (2002) 2647.

[25] A. Kumar, D. K. Avasthi, J. C. Pavin, and P. M. Koinkar, Appl. Phys. Lett.92, (2008) 2219041 - 221904-3.

[26] D. V. Kosynkin, A. L. Higginbotham, A. Sinitskii, J. R. Lomeda, A. Dimiev, B. K. Price, and J. M. Tour, Nature 458, (2009) 872-876.

[27] L. Jiao, L. Zhang, X. Wang, G. Diankov, and Hongjie Dai, Nature 458, (2009) 877-880.

[28] J. C. Delgado, J. M. R. Herrera, X. Jia, D. A. Cullen, H. Muramatsu, Y. A. Kim, T. Hayashi, Z. Ren, D. J. Smith, Y. Okuno, T. Ohba, H. Kanoh, K. Kaneko, M. Endo, H. Terrones, M. S. Dresselhaus, and M. Terrones, Nano Letts. 8, (2008) 2773-2778.

[29]. A. G. C.Marquez, F. J. R. Macias, J. C. Delgado, C. G. E. Gonzalez, F. T. Lopez, D. R. Gonzalez, D. A. Cullen, D. J. Smith, M. Terrones, and Y. I. V. Cantu, Nano Letts. 9, (2009) 15271533.

[30] N. Bajwa, "Phase transformation and fragmentation of fullerene films under ion irradiation" a thesis submitted to Department of Physics, Panjab University, Chandigarh. 
Journal of Nano Research Vol. 10

doi:10.4028/www.scientific.net/JNanoR.10

Structural Modifications of Multiwalled Carbon Nanotubes by Swift Heavy lons Irradiation

doi:10.4028/www.scientific.net/JNanoR.10.1

\section{References}

[1] B.Q. Wei, J.D. Arcy-Gall, P.M. Ajayan and G. Ramanath, Appl. Phys. Lett. 83 (2003) 3581-3583.

doi:10.1063/1.1622781

[2] B. Ni, R. Andrews, D. Jacques, D. Qian, M.B.J. Wijesundara, Y. Choi, L. Hanley, and S.B. Sinnott, J. Phys. Chem. B 105 (2001) 12719-12725.

doi:10.1021/jp0123233

[3] N. Bajwa, A. Ingale, D.K. Avasthi, R. Kumar, A. Tripathi, K. Dharamvir, and V.K. Jindal, Radiation measurement 36 (2003) 737-740.

doi:10.1016/S1350-4487(03)00237-3

[4] A.V. Krasheninnikov, and K. Nordlund, Nuclear Instruments and Methods in Physics Research B 216 (2004) 355-366.

doi:10.1016/j.nimb.2003.11.061

[5] Z. Wang, L. Yu, W. Zhang, Y. Ding, Y. Li, J. Han, Z. Zhu, H. Xu, G. He, Y. Chen, and G. Hu, Phys. Lett. A 324 (2004) 321-325.

doi:10.1016/j.physleta.2004.02.001

[6] Y. Kurokawa, Y. Ohno, S. Kishimoto, T. Okazaki, H. Shinohara, and T. Mizutani, J. Appl. Phys. 43 (2004) (Japan) 5669-5670.

[7] Y. Joon Jung, Y. Homma, R. Vajtai, Y. Kobayashi, T. Ogino, and P.M. Ajayan, Nano letters 4, (2004) 1109-1113.

doi:10.1021/nl049550b

[8] M. Suzuki, K. Ishibashi, K. Toratani, D. Tsuya, and Y. Aoyagi, Appl. Phys. Lett. 81 (2002) 2273-2275.

doi:10.1063/1.1507608

[9] K. Ishibashi, D. Tsuya, M. Suzuki and Y. Aoyagi, Appl. Phys. Lett. 82 (2003) 3307-3309. doi:10.1063/1.1572537

[10] A. Misra, P.K. Tyagi, M.K. Singh, D.S. Misra, J. Ghatak, P.V. Satyam, and D.K. Avasthi, Diamond and Related Materials 15 (2006) 300-303. doi:10.1016/j.diamond.2005.10.021 
[11] H. M. Kim, H.S. Kim, S.K. Park, J. Joo, T.J. Lee, and C.J. Lee, J. Appl. Phys. 97 (2005) 026103-1 - 026103-3.

[12] M. Hulman, V. Skakalova, S. Roth, and H. Kuzmany, J. Appl. Phys. 98 (2005) $024311-$ $1-024311-5$.

[13] U. Ritter, P. Scharff, C. Siegmund, O.P. Dmytrenko, N.P. Kulish, Y.I. Prylutskyy, N.M. Belyi, V. A. Gubanov, L. I. Komarova, S. V. Lizunova, V. G. Poroshin, V. V. Shlapatskayan, and $\mathrm{H}$. Bernas, Carbon 44 (2006) 2694-2700.

doi:10.1016/j.carbon.2006.04.010

[14] F. Banhart, J.X. Li, and A.V. Krasheninnikov, Phys. Rev. B 71 (2005) 241408-1 241408-4.

[15] N. Bajwa, K. Dharamvir, and V. K. Jindal A. Ingale, D.K Avasthi, R. Kumar, A. Tripathi, J. Appl. Phys. 94 (2003) 326-333.

doi:10.1063/1.1581381

[16] N. Bajwa, A. Ingale, D.K Avasthi, R. Kumar, A. Tripathi, K. Dharamvir, and V.K. Jindal, J. Appl. Phys. 104 (2008) 054306-1 - 054306-13.

[17] A.V. Krasheninnikov, F. Banhart, J.X. Li, A. Foster, and R. Nieminen, Phys. Rev. B 72 (2005) 125428-1 -125428-6.

[18] M.S. Dresselhaus, G. Dresselhaus, R. Saito, and A. Jorio, Physics Reports, 409:2, (2005), 47-99.

[19] A. Jorio, R. Saito, J. H. Hafner, C. M. Lieber, M. Hunter, T. McClure, G. Dresselhaus, and M. S. Dresselhaus, Phys. Rev. Lett. 86, $1118-1121$

doi:10.1103/PhysRevLett.86.1118

PMid:11178024

[20] M. Milnera, J. Kürti, M. Hulman, and H. Kuzmany, Phys. Rev. Lett. 84, (2000), 1324 1327.

doi:10.1103/PhysRevLett.84.1324

PMid:11017509

[21] X. Zhao, Y. Ando, L.-C. Qin, H. Kataura, Y. Maniwa, and R. Saito, Physica B:

Condensed Matter, 323:1-4, (2002), 265-266.

[22] S. Costa, E.B. Palen, M. Kruszynska, A. Bachmatiuk, and RJ. Kalenczuk, Materials Science-Poland, 26/2 (2008) 433 -441.

[23] R.J. Nemanich, and S.A. Solin, Phys. Rev. B 20 (1979) 392-401.

doi:10.1103/PhysRevB.20.392 
[24] J. Maultzsch, S. Reich, C. Thomsen, S. Webster, R. Czerw, D.L. Carroll S. M.C. Vieira, P. R. Birkett, and C. A. Rego, Appl. Phys. Lett. 81, (2002) 2647.

doi:10.1063/1.1512330

[25] A. Kumar, D. K. Avasthi, J. C. Pavin, and P. M. Koinkar, Appl. Phys. Lett.92, (2008) 221904-1 - 221904-3.

[26] D.V. Kosynkin, A.L. Higginbotham, A. Sinitskii, J.R. Lomeda, A. Dimiev, B.K. Price, and J.M. Tour, Nature 458, (2009) 872-876.

doi:10.1038/nature07872

PMid: 19370030

[27] L. Jiao, L. Zhang, X. Wang, G. Diankov, and Hongjie Dai, Nature 458, (2009) 877-880. doi:10.1038/nature07919

PMid:19370031

[28] J.C. Delgado, J. M.R. Herrera, X. Jia, D. A. Cullen, H. Muramatsu, Y.A. Kim, T. Hayashi, Z. Ren, D. J. Smith, Y. Okuno, T. Ohba, H. Kanoh, K. Kaneko, M. Endo, H. Terrones, M. S. Dresselhaus, and M. Terrones, Nano Letts. 8, (2008) 2773-2778. doi:10.1021/nl801316d

PMid:18700805

[29] A. G. C.Marquez, F. J. R. Macias, J. C. Delgado, C. G. E. Gonzalez, F. T. Lopez, D. R. Gonzalez, D.A. Cullen, D.J. Smith, M. Terrones, and Y.I. V. Cantu, Nano Letts. 9, (2009) 1527-1533.

doi:10.1021/nl803585s

PMid: 19260705

[30] N. Bajwa, "Phase transformation and fragmentation of fullerene films under ion irradiation" a thesis submitted to Department of Physics, Panjab University, Chandigarh. 